\title{
Morte Encefálica, Cuidados ao Doador de Órgãos e Transplante de Pulmão*
}

\author{
Brain Death, Multiorgan Donor and Lung Transplantation
}

\author{
Fernando D'Império ${ }^{1}$
}

\section{RESUMO}

JUSTIFICATIVA E OBJETIVOS: O transplante de órgãos é aceito como uma opção para doença orgânica terminal, em pacientes bem selecionados. Este é o resultado de grandes avanços nos campos da Imunologia, da Tecnologia da Terapia Intensiva e da Farmacologia. Entretanto, os sistemas de transplantes são, atualmente, vítimas de seu próprio sucesso, à medida que as listas de espera se alongam, em contraste com a disponibilidade de órgãos, que permanece estável, acarretando crescente número de mortes nestas filas de espera. A comunidade de transplante respondeu a esta situação revendo os critérios de aceitabilidade de doadores e desenvolvendo novas estratégias na obtenção de órgãos (como nos casos de captação após a parada circulatória).

CONTEÚDO: Entretanto muito se evoluiu na compreensão da fisiopatologia da morte encefálica e suas conseqüências visando a melhor manutenção do doador potencial de múltiplos órgãos e utilização destes. Este artigo procurou discutir os pontos de maior interesse na manutenção clínica do paciente com morte encefálica, correlacionando com a sua fisiopatologia e apontando os pontos considerados de maior relevância específica como a depleção de aminas vasoativas e a manutenção

1. Professor Associado de Cirurgia Torácica da Escola Médica de Pós-Graduação da PUC-Rio e Assistente Doutor da Divisão de Cirurgia Torácica do IDT-UFRJ

*Estudo realizado em Toronto, Universidade de Toronto, Canadá.

Apresentado em 07 de dezembro de 2006

Aceito para publicação em 03 de março de 2007

Endereço para correspondência:

Dr. Fernando D'Império.

Av Armando Lombardi 1000. Bloco 1 - Sala 313

22640-000 Rio de Janeiro, RJ

Fone: (21) 2492-8210 / Fax: 2493-0255

E-mail: fdimperio@globo.com

(C)Associação de Medicina Intensiva Brasileira, 2007 dos sistemas cardiorrespiratório, distúrbios endócrinos e hidroeletrolítcos. Observam-se também algumas recomendações quanto ao aproveitamento e de órgãos específicos (coração, pulmão, rim e fígado).

CONCLUSÕES: Com o aumento significativo das listas de espera por órgãos e a escassez de órgãos disponíveis leva-nos a um esforço para aprimorar as técnicas existentes de captação e preservação, assim como desenvolver novas medidas para seu aproveitamento de forma a reduzir a mortalidade nas filas de espera que são sempre uma sombra nos programas de transplantes.

Unitermos: doadores de tecidos, pulmão, transplante de órgãos

\section{SUMMARY}

BACKGROUND AND OBJECTIVES: Organ transplantation is now an accepted option for end stage organ disease in well selected patients. This position is a result of great advances in the field of immunology, critical care medicine and pharmacology. However, organ transplantation is now suffering from its own success as the number of patients in waiting lists is dramatically increasing the same is not happening with organ availability results in increasing number of mortalities while waiting for transplantation. Transplant community responses to this situation consist of reviewing the criteria for organ acceptability and developing new strategies to get organs as the called non-heart beating organ donors.

CONTENTS: However the physiopathology of brain death and its consequences are now better understood helping in such patients' management. The purpose of this review is to help to identify the most important clinical and therapeutic aspects related to its physiopathology as depletion of vasoactives substances and its importance in the management of cardio and respiratory systems. We also discuss endocrine and hidroelectrolytes disturbances. Organ specific data are also focused in order to offer a whole view of donor management. 
CONCLUSIONS: It is important to observe that new technologies will be available in the near future to diminish the low rate between organ availability and organ waiting patients.

In conclusion, with the raising numbers in transplant waiting lists and scarce resources of organs make us believe that we have to improve the management of multi organ donors and the preservation technology in order to reduce the mortality in such waiting lists.

Key Words: brain death, lung, organ transplantation, tissue donors

\section{INTRODUÇÃO}

O transplante de órgãos tornou-se uma opção de excelência no tratamento da falência terminal de órgãos em pacientes bem selecionados. Tal posição foi conquistada depois de grandes avanços nas áreas de Terapia Intensiva, da Imunologia e da Farmacologia. Entretanto o transplante de órgãos tornou-se vítima de seu próprio sucesso, a medida que o número de pacientes aguardando por um transplante excedeu em muito a disponibilidade de órgãos para doação e verificaram-se crescentes taxas de mortalidade na fila de espera. A comunidade de transplante vem respondendo a esta carência de órgãos com a flexibilização dos critérios clínicos de inclusão de doadores de órgãos (doadores marginais, estendidos ou não ideais ou seja, doadores que estão fora dos critérios ótimos para a doação), com a aceitação de doadores após a parada cardíaca e a chamada doação intervivos ${ }^{1}$. Uma outra modalidade de transplantes ainda em fase laboratorial são os xenotransplantes.

O incremento do número de doadores e de efetivas doações envolve melhor compreensão da morte encefálica, seus processos fisiopatológicos, sua identificação e as estratégias envolvidas no equilíbrio clínico do doador. Um programa de transplante de um determinado órgão não deve ser visto de forma isolada. Suas bases organizacionais, suas necessidades, seus modos de atuação e composição de equipes multiprofissionais os tornam muito semelhantes. A participação da comunidade leiga se faz essencial à medida que se constitui na origem dos doadores. Campanhas de educação geral sobre transplantes e seus conceitos envolvidos, o esclarecimento de dúvidas e o combate à mitos devem ser constantes. Deve-se observar que mesmo os próprios profissionais de saúde devem ser alvo de campanhas de esclarecimento e treinamento.

O objetivo deste estudo foi fazer uma proposta de abor- dagem do tema morte encefálica e os cuidados avançados com o doador. Procurou-se transmitir a experiência adquirida durante os últimos três anos no Canadá, trabalhando no "Toronto General Hospital", como cirurgião de transplante de pulmão e captação de órgãos, em um dos mais avançados e movimentados centros mundiais de transplante múltiplo de órgãos, em especial, o pulmão que teve seu primeiro caso aí realizado.

\section{O CONCEITO DE MORTE ENCEFÁLICA}

O conceito de morte encefálica iniciou-se em 1959 com a descrição do "coma depassé". Progressos na Terapia Intensiva proveram uma capacidade de manutenção e suporte, de modo a manter as funções básicas do organismo por um período determinado a despeito de um encéfalo não funcionante. Esta condição passou a ser conhecida, recentemente como "morte encefálica"2. Entretanto com o recrudescimento de questões éticas, morais, religiosas e legais, após o primeiro transplante cardíaco realizado na África do Sul, em 1968, a "Harvard Medical School" definiu critérios para a "morte encefálica" e constituiu a base médico-legal para a utilização de órgãos destes pacientes. Este trabalho foi importante por oferecer uma definição conceitual sobre morte encefálica, estabelecer critérios diagnósticos para que esta condição fosse reconhecida e por redefinir o estado de morte, apesar do contínuo funcionamento de outros órgãos ${ }^{3}$.

A morte encefálica foi conceituada como o déficit estrutural e/ou funcional do encéfalo como órgão de função integradora e crítica ao organismo humano (Harvard, 1969)4. A seqüência de eventos durante esta fase vem demonstrando sua importância sobre o processo de transplante propriamente dito e um claro fator de risco para o receptor. Tais observações são comprovadas quando se observam transplantes com identidade HLA e nos casos de doação intervivos onde o tempo de isquemia é otimizado 5 .

A morte encefálica é um processo complexo que altera a fisiologia de todos os sistemas orgânicos. Recentemente foi reconhecido que ela envolve uma série de perturbações neuro-humorais cíclicas que incluem alterações bioquímicas e celulares que conduzem a disfunção múltipla de

órgãos repercutindo na qualidade do órgão transplantado. Tanto as alterações iniciais quanto as tardias influem na viabilidade dos órgãos ao comprometer sua perfusão, aumentando a lesão isquêmica (Figura 1). O processo de morte encefálica inicia-se com o aumento de pressão intracraniana (PIC) devido a expan- 


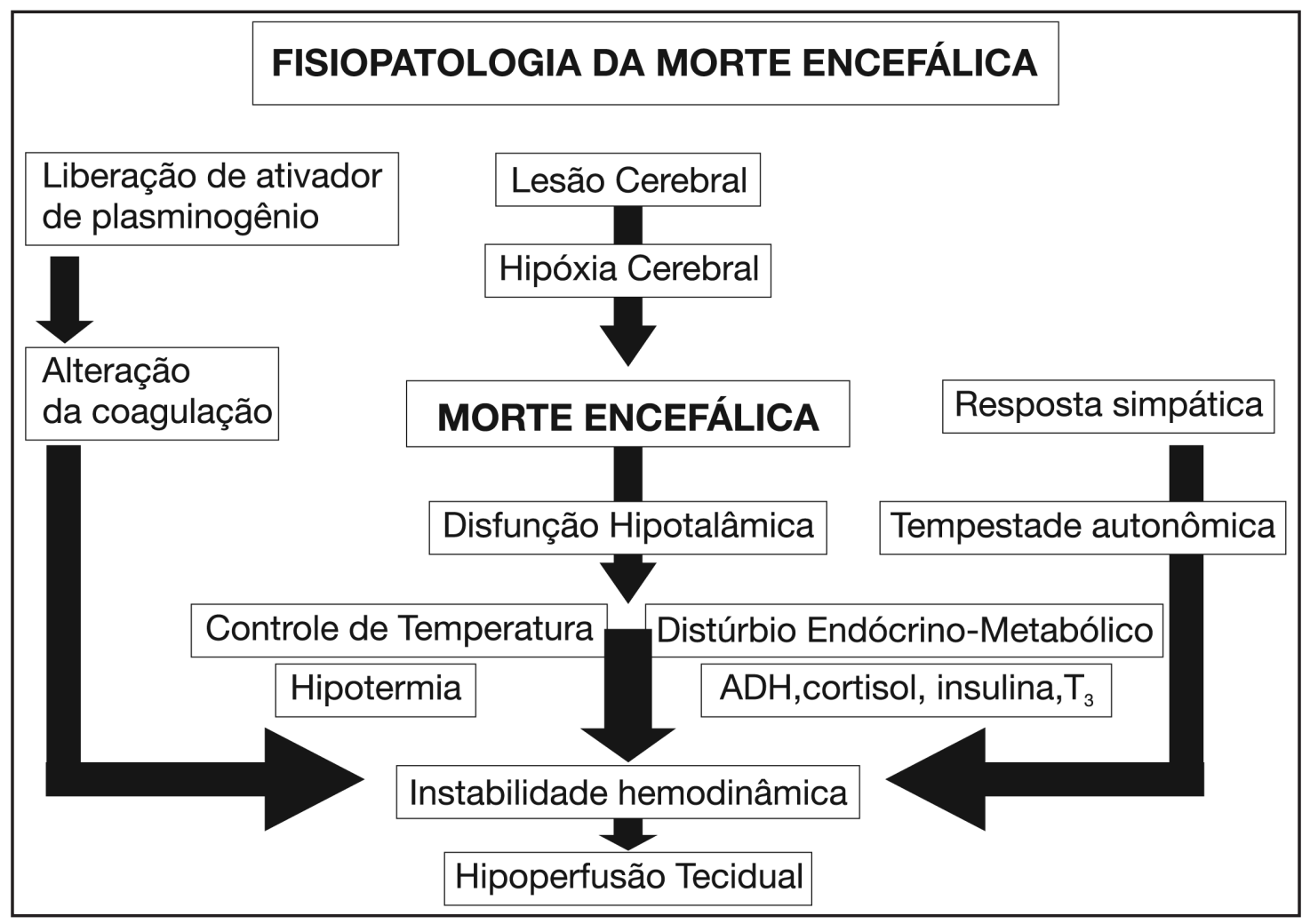

Figura 1 - Esquema Demonstrando os Múltiplos Processos Envolvendo a Fisiopatologia da Morte Encefálica.

são volumétrica do conteúdo intracraniano. Durante esta expansão o conteúdo liqüórico é drenado e o retorno venoso é comprometido elevando progressivamente a PIC. A hipóxia celular e o edema contribuem ainda para este aumento. Este processo culmina com a herniação transtentorial do tronco cerebral pelo forâmen magno que bloqueia por completo a via de única saída, elevando a PIC até o momento que interrompe completamente a circulação arterial encefálica seguindo-se, então, a morte encefálica² .

Alterações hemodinâmicas são observadas durante estas alterações. Na fase inicial ocorre diminuição da pressão arterial e da freqüência cardíaca devida a atividade parassimpática secundária à elevação da PIC.

Quando a isquemia atinge o tronco encefálico ocorre ativação simpática secundária ao acometimento do centro vagal cárdiomotor na ponte (medula oblongata) com intensa elevação da pressão arterial decorrente da descarga adrenérgica, o reflexo de Cushing. Esta fase é conhecida como "tempestade adrenérgica" e tem a duração desde minutos a horas ${ }^{2}$. Estes eventos traduzem a tentativa do organismo de restabelecer a comprometida circulação cerebral. Esta tentativa é mais intensa quanto mais agudo for o processo de morte encefálica. A presença de maior concentração de catecolaminas no coração independente dos mecanismos descritos, pode ser muito importante na eventual função do enxerto cardíaco. Durante esta fase encontra-se desvio do metabolismo aeróbio às vias de metabolismo anaeróbico, aumento do cálcio intracelular, diminuição dos níveis de ATP, acúmulo de lactato, depleção de glicogênio hepático, geração de radicais livres de oxigênio e alterações das membranas mitocondriais ${ }^{1}$.

Progressão distal da isquemia leva a desativação simpática com declínio significativo da resistência vascular periférica com hipoperfusão tecidual.

$A$ isquemia do tronco cerebral é seguida de intensa redução dos hormônios anteriores e posteriores da hipófise, especialmente seus lobos anteriores, decorrentes do comprometimento do eixo hipotálamo-hipofisário 1-3,6.

A redução dos níveis do hormônio antidiurético (ADH) é encontrada em cerca de $90 \%$ dos casos e está envolvido na manutenção do equilíbrio hemodinâmico e na estabilidade cardiovascular nesta fase. Sua manifestação mais óbvia é a diabetes insipidus. Porém, este hormônio tem ação sinérgica com as catecolaminas na manutenção do tônus vasomotor e da integridade endotelial ${ }^{1-3,6}$. Distúrbio tireoidiano também está presente. O TSH encontra-se diminuído, assim como os níveis de $\mathrm{T}_{3}$. Este nível de hormônio encontra-se diminuído às custas da 
elevação de metabólito do $T_{4}$ pouco ativo, o $r T_{3}$. Além disso, está presente uma redução da expressão periférica de receptores do $T_{3}$. É discutível a relação dos diminutos níveis de $\mathrm{T}_{3}$ com a redução do metabolismo oxidativo e o grau de distúrbio miocárdico².

Os hormônios pancreáticos também se encontram alterados durante esta fase. Níveis elevados de insulina e peptídeo $\mathrm{C}$ são demonstrados enquanto o glucagon está normal ${ }^{1}$.

Os pulmões são órgãos altamente vulneráveis às alterações hemodinâmicas descritas. Durante o período de intensa descarga adrenérgica o sangue é redistribuído e ocorre um aumento do retorno venoso ao ventrículo direito o qual aumenta rapidamente seu débito, aumentando o fluxo pulmonar. Simultaneamente a pressão de átrio esquerdo está elevada devido a vasoconstrição periférica intensa de tal forma que a pressão hidrostática capilar é muito aumentada promovendo ruptura de capilares com edema intersticial e hemorragia alveolar $^{2}$. Tais alterações manifestam-se com o desequilíbrio ventilação perfusão e hipoxemia. Deve-se combater estas áreas de atelectasia para diminuir o shunt e estimular secreção de surfactante que se encontra diminuída no período pós-transplante ${ }^{7-9}$.

Por uma variedade de razões incluindo a lesão original e os transtornos decorrentes da morte encefálica, distúrbios eletrolíticos estão sempre presentes. Hiponatremia, hipocalemia, hipofosfatemia, hipomagnesemia e hipocalcemia exigem reposição imediata. A hiperglicemia normalmente é decorrente de reposição hídrica deficiente; entretanto, existem alterações dos hormônios envolvidos em sua homeostase além de associado quadro de insuficiência adrenal ${ }^{10-12}$. A hipotermia é quase que universal e contribui para depressão miocárdica, anormalidades de coagulação, hipertensão pulmonar e instabilidade hemodinâmica ${ }^{1,2,13}$.

Em 1998, foi demonstrado que o estresse decorrente deste período conduz a graves lesões orgânicas, secundária à eliminação de citocinas e quimiocinas. Tais proteínas seriam responsáveis por órgãos "ativados" e menos viáveis. Um influxo leucocitário ao órgão pode ser demonstrado e atribuído a essa secreção de proteínas. Este aumento de imunogenecidade parece ser proporcional ao tempo de morte encefálica ${ }^{13,14}$. Grosseiramente, entende-se por ativado ao estado induzido por certas proteínas onde a expressão de moléculas de adesão (ICAM-molécula de adesão intercelular, VCAMmolécula de adesão de células vasculares) e antígenos de histocompatibilidade leucocitária classe II DR estão aumentados nas membranas do endotélio e leucócitos, tornando os mais imunogênicos e sujeitos aos processos de rejeição imunológica no receptor ${ }^{15,16}$.

\section{SELEÇÃO DO DOADOR DE ÓRGÃOS}

Considerações sobre o paciente com morte encefálica se fizeram necessárias para se decidir sobre a possibilidade de um paciente tornar-se doador de órgãos. Quase que $80 \%$ dos casos são secundários a lesões traumáticas e acidentes vasculares encefálicos. Outras causas comuns são tumores do sistema nervoso central e uso de drogas e intoxicações ${ }^{1}$.

A avaliação de um possível doador de órgãos e tecidos iniciam-se com cuidadosa revisão da história clinica e social, exame físico com atenção especial à sinais de malignidade, trauma e comportamento de risco. As contra-indicações ou critérios de exclusão para a doação de órgãos e tecidos estão apresentados no quadro 1. Exames laboratoriais sorológicos (Quadro 2) são solicitados após o consentimento para doação ter sido assinado. Estabelece-se rotina de monitorização laboratorial de acordo com o proposto na tabela $1^{1,12,17-19}$.

Quadro 1 - Critérios Gerais de Exclusão para Doação de Órgãos e Tecidos

\begin{tabular}{l}
\hline Sepse não tratada \\
Tuberculose em atividade \\
Infecção por HIV \\
Encefalite viral \\
Hepatite viral (existem exceções) \\
Síndrome de Guillain-Barré \\
Uso de drogas ilícitas por via venosa \\
História de malignidade
\end{tabular}

Adaptado do Programa de Transplante de Pulmão de Toronto, Universidade de Toronto (2006).

Quadro 2 - Exames Sorológicos Solicitados de Rotina para o Potencial Doador.

\begin{tabular}{ll}
\hline Sífilis - VDRL & Venereal Disease Research Lab \\
Anti-HTLV I / II & Anticorpo antilinfócito T linfotrófico vírus humano \\
Anti-HIV I / II & Anticorpo anti Vírus da imunodeficiência humano \\
HBsAg & Antígeno de superfície do vírus da Hepatite B \\
HBcAb & Anticorpo contra o antígeno core da Hepatite B \\
Anti-HCV & Anticorpo contra vírus da hepatite C \\
Anti-CMV & Anticorpo contra citomegalovírus \\
Anti-EBV & Anticorpo contra o vírus Epstein-Barr \\
Toxoplasmose & Anticorpo contra toxoplasma gondii \\
Observação & Teste sorológico da mãe no caso de doador \\
& menor de 18 meses ou no caso de aleitamento \\
& materno há menos de 18 meses. \\
& Atenção ao fator de hemodiluição e sua reper- \\
& cussão na aferição dos resultados laboratoriais. \\
\hline
\end{tabular}

Adaptado do Programa de Transplante de Pulmão de Toronto, Universidade de Toronto (2006) 
Tabela 1 - Monitorização do Doador Múltiplo de Órgãos.

\begin{tabular}{|c|c|c|}
\hline Eletrólitos [Na, K] & $4 / 4 \mathrm{hr}$ & \\
\hline Uréia, creatinina & $4 / 4 \mathrm{hr}$ & \\
\hline Glicose & $4 / 4 \mathrm{hr}$ & \\
\hline Hemograma completo & $4 / 4 \mathrm{hr}$ & \\
\hline PTT, TAP, INR & $4 / 4 \mathrm{hr}$ & \\
\hline Amilase & $4 / 4 \mathrm{hr}$ & \\
\hline Testes de função hepática & $4 / 4 \mathrm{hr}$ & $\begin{array}{l}\text { Bilirrubina, TGO, TGP, } \\
\text { GGT, LDH, FAlc }\end{array}$ \\
\hline Gasometria arterial & $4 / 4 \mathrm{hr}$ & Intestino, pulmão \\
\hline Troponina, CK, CK-MB & $4 / 4 \mathrm{hr}$ & Coração \\
\hline $\begin{array}{l}\text { PVC, PAD, PAP, PAPE, IC, RVS, } \\
\text { RVP }\end{array}$ & & $\begin{array}{l}\text { Coração, pulmão, in- } \\
\text { testino }\end{array}$ \\
\hline Depuração de creatinina & & Rim \\
\hline Altura / peso & & $\begin{array}{l}\text { Fígado, intestino, co- } \\
\text { ração }\end{array}$ \\
\hline Diâmetro abdominal & & Fígado \\
\hline Anatomia patológica & & Rim, fígado, pulmão \\
\hline Cultura urina, escarro e sangue & & \\
\hline Radiografia de tórax AP & \multirow[t]{3}{*}{$12 / 12 \mathrm{hr}$} & Coração, pulmão \\
\hline Eletroencefalograma & & Coração \\
\hline Ecocardiografia 2D & & $\begin{array}{l}\text { Coração, pulmão, in- } \\
\text { testino }\end{array}$ \\
\hline Coronariografia & & Coração \\
\hline Balanço hídrico & $1 / 1 \mathrm{hr}$ & Coração, pulmão \\
\hline СРТ & & Pulmão \\
\hline $\begin{array}{l}\text { Recrutamento pulmonar }(30 \\
\text { mmHg por } 30 \text { seg) } A B G \text { após } 10 \\
\text { min em PEEP } 5 / F_{i O} 1,0\end{array}$ & $3 / 3 \mathrm{hr}$ & Pulmão \\
\hline
\end{tabular}

PVC: pressão venosa central, PAD: pressão de átrio direito, PAP: pressão de artéria pulmonar, PAPE: pressão de artéria pulmonar encunhada, IC: índice cardíaco, RVS: resistência vascular sistêmica, RVP: resistência vascular pulmonar, CPT : capacidade pulmonar total.

Adaptado do Programa de Transplante de Pulmão de Toronto, Universidade de Toronto (2006)

\section{CRITÉRIOS DE SELEÇÃO ESPECÍFICOS PARA A DOAÇÃO/CAPTAÇÃO DOS PULMÕES.}

Os critérios específicos para o aproveitamento dos pulmões de eventual doador devem ser considerados. Resumidamente são: classificação sangüínea, o tamanho do órgão (capacidade total prevista), história de tabagismo, história pregressa de doença pulmonar, função pulmonar (gases arteriais), aspecto radiológico, achados broncoscópicos e inspeção cirúrgica. Tipicamente um doador de pulmão corresponde aos critérios estabelecidos para um doador ideal (Tabela 2). A flexibilização dos critérios de aceitação (doador estendido, não ideal ou marginal) é uma contra medida à escassez de órgão frente à crescente lista de espera por doadores de pulmão ${ }^{10,20,21}$. A idade do doador não consiste em fator de risco isoladamente, porém se correlaciona negativamente com a duração do período de isquemia, onde se verificou aumento da mortalidade ${ }^{22}$.
Tabela 2 - Avaliação do Doador de Pulmão.

\begin{tabular}{|c|c|c|}
\hline & Doador Ideal & Doador Estendido \\
\hline Idade & $<55$ anos & $>55$ anos \\
\hline Grupo sangüíneo & Identidade & Compatibilidade \\
\hline Tabagismo & Não fumante (<20??) & $<30$ maços/ano \\
\hline Radiografia de tórax & Normal & Anormal \\
\hline Trauma torácico & Ausente & Presente \\
\hline Broncoscopia & Normal & Anormal \\
\hline $\begin{array}{l}\mathrm{PaO}_{2}\left(\mathrm{FiO}_{2}, 1,0, \mathrm{PEE}\right. \\
\left.5 \mathrm{cmH}_{2} \mathrm{O}\right)\end{array}$ & $>300 \mathrm{mmHg}$ & $>300 \mathrm{mmHg}$ \\
\hline $\begin{array}{l}\text { Cirurgia torácica } \\
\text { anterior }\end{array}$ & não & $?$ \\
\hline $\begin{array}{l}\text { Secreção purulenta } \\
\text { à broncoscopia }\end{array}$ & Ausente & Presente \\
\hline Gram do escarro & Negativo & Positivo \\
\hline $\begin{array}{l}\text { Sinais de aspiração } \\
\text { gástrica }\end{array}$ & Não & Não \\
\hline
\end{tabular}

Adaptado do Programa de Transplante de Pulmão de Toronto, Universidade de Toronto (2006)

A utilização dos gases arteriais como critério de seleção merece consideração acerca de utilidade pontual. Este método traduz apenas a boa condição de troca gasosa naquele instante, porém não constitui em indicador para um bom enxerto. É comum encontrar bons índices de oxigenação arterial (alta $\mathrm{PaO}_{2} / \mathrm{FiO}_{2}$ ) em pulmões inadequados para transplante, como no caso de broncoaspiração de conteúdo gástrico.

A radiografia de tórax é essencial para a avaliação do doador. Procuram-se condições óbvias que precludiriam a utilização posterior do órgão como doenças pulmonares prévias. A presença de infiltrados, edemas e atelectasias devem ser avaliadas individualmente, em correlação com todos os achados de história clínica e demais exames complementares, incluindo os broncoscópicos .A presença de secreção purulenta nas vias aéreas distais não contra-indica o transplante, o mais importante é a quantidade segmentos acometidos e o volume de drenado. Convém utilizar-se de cobertura antibiótica profilática até a elucidação microbiológica, incluindo a controversa antibioticoterapia profilática no doador já que é muito comum o exame bacteriológico de secreção respiratória demonstrar bactérias (estudo pela coloração de Gram) ${ }^{1}$.

Como já mencionado o tamanho do órgão do receptor deve ser semelhante ao do doador. A capacidade total pulmonar prevista é o método mais utilizado para assegurar que um órgão é grande demais para se adequar a caixa torácica do receptor seja transplantado, assim como o inverso, método de cálculo demonstrado na tabela 3. No caso de um órgão sub-dimensionado para o receptor (ainda mais importante nos transplante lo- 
bares) criam problemas de câmara pleural residual e dificuldades de manutenção da expansibilidade do enxerto. No caso de um enxerto grande para a cavidade torácica tem-se a possibilidade de cirurgia redutora de volume para evitar atelectasia pós-operatória e o tamponamento cardíaco $23-25$.

Tabela 3 - Cálculo da Capacidade Pulmonar Total (CPT).

\begin{tabular}{lcc}
\hline CPT & Sexo Feminino & Sexo Feminino \\
\hline Roberts, 1992 & $7,956 \times(\mathrm{h})-6,948$ & $7,107 \times(\mathrm{h})-6,435$ \\
Grimby, 1963 & $6,92 \times(\mathrm{h})-0,017 \times(\mathrm{kg})$ & $6,71 \times(\mathrm{h})-0,015 \times(\mathrm{kg})$ \\
& $-4,30$ & $-5,77$ \\
Goldman, 1959 & $0.094 \times(\mathrm{h})-0.015 \times(\mathrm{a})$ & $0.079 \times(\mathrm{h})-0.008 \times(\mathrm{a})$ \\
& $-9,167$ & $-7,49$ \\
\hline
\end{tabular}

h - altura em metros; kg - peso em quilos, a - idade em anos.

A relação entre tabagismo e doença pulmonar é bem conhecida. Ao se flexibilizarem os critérios de aceitabilidade no tocante ao tabagismo ressalta-se a possibilidade de se transplantarem pulmões acometidos de hiper-reatividade brônquica, hipersecreção, doença distal de vias aéreas e mesmo tumores já presentes ou que virão a se manifestar com a imunossupressão ${ }^{26-28}$. Ainda não está comprovado se a asma brônquica induz a remodelação de vias aéreas, o que pode comprometer a função do enxerto de curto e de longo prazo ${ }^{16}$. Outro dois aspectos que ainda merecem melhor esclarecimento são o tempo de ventilação mecânica do doador e a causa da sua morte ${ }^{22}$.

O exame broncoscópico encontra-se normal em apenas um terço dos casos. Esta anormalidade deve ser encarada com muito cuidado, pois refletirá sobre a função do órgão do eventual receptor Sinais de infecção (edema e hiperemia de mucosa brônquica, presença secreção purulenta (localização proximal ou distal, quantidade, número de segmentos acometidos), drenagem contínua) e aspiração de conteúdo gástrico devem ser pesquisados com cuidado. O risco de deterioração da troca gasosa é bastante grande, diante da presença de comprometimento parenquimatoso por infecção. Não devem ser transplantados pulmões com sinais de aspiração. A realização de lavado broncoalveolar para estudo bacteriológico será bastante útil para guiar a antibioticoterapia do receptor no pós-operatório imediato. A utilização de órgãos marginais deve ser avaliada em conjunto com o estado do pulmão do doador, a doença básica do receptor e a gravidade de seu caso e o procedimento proposto para o transplante (bilateral, unilateral ou lobar) além da compatibilidade de tamanhos ${ }^{29-34}$.

\section{TRATAMENTO DO POTENCIAL DOADOR DE ÓR- GÃOS}

Considerações sobre o potencial doação de órgãos devem começar precocemente em todo paciente em tratamento intensivo, pois no momento da morte encefálica há inversão de prioridades para a manutenção de órgãos para transplante a despeito da lesão neurológica, momento em que o conhecimento da fisiopatologia se faz essencial. É imperativo o contínuo envolvimento do pessoal de terapia intensiva já que isto refletirá sobre os receptores. Neste contexto, monitorização específica se faz necessária, muitas vezes ainda mais agressiva, pois tal procedimento favorece o aproveitamento de órgãos ${ }^{35}$.

A manutenção do potencial doador inclui o desafio da estabilidade hemodinâmica. Disritmias atrial e ventricular podem estar presentes e devem ser tratadas e não constitui contra-indicação a utilização cardíaca por si só. Anormalidade de motilidade de parede ventricular é freqüente, especialmente a do ventrículo direito, assim como algum grau de movimento paradoxal do septo interventricular. Acrescenta-se que cerca de $87 \%$ dos portadores de morte encefálica evoluem com disritmia cardíaca maligna em menos de 72 horas. A importância da falência ventricular direita é avaliada no peri-operatório observando-se a pronta recuperação à inspeção pelo cirurgião. A hipovolemia é uma manifestação universal e deve ser tratada, não deve ser subestimada com a apreciação de edema pulmonar. Objetivos gerais na manutenção cardiovascular incluem pressão arterial sistólica acima de $90 \mathrm{mmHg}$, freqüência cardíaca em torno de $100 \mathrm{bpm}$ e pressão venosa central entre 8 e $10 \mathrm{mmHg}$. Tais valores devem ser atingidos com reposição volumétrica e aminas, se necessárias. Inicialmente preconiza-se a infusão de solução fisiológica de $500 \mathrm{~mL} / 15 \mathrm{~min}$ seguida de reposição das perdas urinárias. Possivelmente tal volume não é tolerado pelo potencial doador de pulmão, no qual restringe-se a $100 \mathrm{~mL} / \mathrm{h}$ de solução fisiológica. No caso de simpatectomia decorrente da morte encefálica o uso de vasoconstritor está indicado. A vasopressina parece ser o mais efetivo e de menor associação com disritmias cardíacas. Chama-se atenção para ocasionais situações de denervação funcional do coração onde a resposta à atropina está comprometida. Nestas ocasiões os cronotrópricos (dopamina ou isoproterenol) ou mesmo marca-passo temporário deve ser utilizado'.

Todos os possíveis doadores exigem ventilação mecâ- 
nica. O objetivo é a manutenção da saturação arterial de oxigênio superior a $90 \%$ com pressão parcial arterial superior a $60 \mathrm{mmHg}$. Procurar manter a fração inspiratória em torno de $40 \%$ com pressões inspiratórias baixas. Normalmente utilizam-se respiradores ciclando a pressão como opção protetora ao barotrauma. Procurar não ventilar o potencial doador com altas pressões máximas (PIP), altos volume-corrente (entre 6 e 8 $\mathrm{mL} / \mathrm{kg})$ e alto PEEP $\left(<7 \mathrm{cmH}_{2} \mathrm{O}\right)$.

As anormalidades hidroeletrolíticas são comuns. As mais freqüentes são alcalose respiratória, hipocalemia, hipercalemia, hipernatremia, hipofosfatemia e acidose metabólica. Diabetes insipidus é a causa mais comum de hipernatremia. A reposição hídrica deve seguir ao cálculo do déficit de água livre.

A alcalose é o resultado do tratamento ventilatório para combate a elevação da PIC. Pode acarretar disfunção cardíaca potencializada pelas alterações do cálcio, fosfato e magnésio. Acidose metabólica não apenas indica hipoperfusão tecidual, mas sinaliza possível disfunção cardíaca associada. Aumento do anion gap, acúmulo de lactato são indicativos de baixa perfusão tecidual ${ }^{17}$.

A suplementação com corticóides são controversas. Como já descrito, o estado de morte encefálica envolve uma progressão do processo inflamatório. A utilidade do corticóide nesta situação foi demonstrada por Follete, em 1998, onde se constatou melhora da oxigenação e melhor aproveitamento dos pulmões disponíveis. Preconiza-se assim, a suplementação única com $1 \mathrm{~g}$ de metilprednisolona, por via venosa $(15 \mathrm{mg} / \mathrm{kg})^{36}$. A coagulopatia deve ser considerada e tratada em todos os doadores. Plaquetas, plasma fresco ou crioprecipitado, freqüentemente são necessários e devem ser administrados prontamente ${ }^{37,38}$.

Protocolo da UNOS - United Network for Organ Sharing para cuidados com doador cardiotorácico consiste em:

1. Hemodinâmica:

a. Ecocardiograma para todos;

b. Cateter de artéria pulmonar para os dependentes de aminas ou fração de ejeção $(E F)<45 \%$;

- Pressão venosa central (PVC) $<12$ mmHg;

- Pressão capilar pulmonar encunhada (PCWP) $<12$ $\mathrm{mmHg}$.

- Resistência vascular sistêmica (SVR) 800-1200 dybe/ $\mathrm{sec} / \mathrm{cm} 5$.

- Índice cardíaco > 2,5 L/min/m².

- Índice LVSVI > 15;

- Dopamina $<10 \mu \mathrm{g} / \mathrm{kg} / \mathrm{min}$.
2. Balanço hidroeletrolítico:

a) $\mathrm{Na}<150 \mathrm{mEq} / \mathrm{dL}$

b) $\mathrm{K}>4 \mathrm{mEq} / \mathrm{dL}$

c) Leve hiperventilação $\left(\mathrm{pCO}_{2} 30\right.$ a $\left.35 \mathrm{mmHg}\right)$

d) Reanimação;

e) Preferência aos colóides:

- Albumina, se PT e PTT estiverem normais;

- Plasma fresco, se alterados;

- Concentrado de hemácias, mantendo a PCWP 8 a 12 $\mathrm{mmHg}$ e $\mathrm{Hb}>10 \mathrm{~g} / \mathrm{dL}$.

3. Ventilação:

a) Volume-corrente de 10 a $15 \mathrm{mmHg}$;

b) Pressão máxima da via aérea $<30 \mathrm{mmHg}$;

c) $\mathrm{pCO}_{2} 30$ a $35 \mathrm{mmHg}$.

4. Reposição Hormonal:

a) Triiodotironina $\left(T_{3}\right): 4 \mu \mathrm{g}$ em bolus, $3 \mu \mathrm{g} / \mathrm{h}$ em infusão contínua ou tetra-iodotironina $(20 \mu \mathrm{g})$ seguido de 10 $\mu \mathrm{g} / \mathrm{h}$, ambos por via venosa;

b) Vasopressina: $1 \mathrm{U}$ em bolus; 0,5 a $4 \mu \mathrm{g} / \mathrm{h}$, por via venosa (manter SVR 800-1200);

c) Metilprednisolona: $15 \mathrm{mg} / \mathrm{kg}$ em bolus (repetir a cada 24 h);

d) Insulina: infusão contínua com no mínimo $1 \mathrm{U} / \mathrm{h}$, mantendo a glicose de 120 a 180 mg/dL.

\section{RECOMENDAÇÕES GERAIS:}

\section{Monitorização e Suporte Hemodinâmico}

A deterioração da função cardiovascular associada com a hipertensão intracraniana variará com a velocidade da elevação da PIC, tempo após a herniação e etiologia da lesão cerebral ${ }^{3}$. O suporte cardiovascular hemodinâmico pressupõe euvolemia e é traduzido por um conjunto de aferições, em que nenhum dado isolado deve dirigir o tratamento. Se aceita que a escalada de suporte seja acompanhado por uma escalada na monitorização. São parâmetros de monitorização aceitos: linha arterial e linha central.

São alvos hemodinâmicos: PAM > 70 mmHg; PAS > 100 $\mathrm{mmHg}$; FC > 60bpm e < 120 bpm e PVC 6 a $10 \mathrm{mmHg}$. Manutenção da pressão arterial (PAS $>100 \mathrm{mmHg}$, PAM $>70 \mathrm{mmHg})^{38-43}$. Devido ao risco de deterioração súbita após a morte encefálica, os agentes de curta duração são preferíveis. A hipertensão arterial durante o uso de vasopressores ou inotrópicos tem correção preferencialmente com o ajuste das doses. Caso os anti-hipertensivos se façam necessários, preferir a nitroglicerina (evitar o nitroprussiato pela síndrome de roubo coronariano) 
e/ou labetalol ou esmolol 100 a $500 \mu \mathrm{g} / \mathrm{kg}$, em bolus por via venosa, 100 a $300 \mu \mathrm{g} / \mathrm{kg} / \mathrm{min}^{39-43}$.

Nos pacientes com alguma instabilidade hemodinâmica sugere-se a monitorização da saturação venosa central ou mista. Ajustes devem ser realizados com objetivo de mantê-la acima de $60 \%$. Ressalte-se que a tendência deve ser mais valorizada que uma medição isolada ${ }^{44}$.

Recomenda-se a monitorização do lactato sérico em que qualquer anormalidade deve ser investigada e corrigida ${ }^{41,42,44}$.

A cateterização de artéria pulmonar não é preconizada de rotina. Apesar da tendência a diminuição do SwanGanz em Terapia Intensiva, o paciente em morte encefálica é um caso bem distinto, onde se encontra total desarranjo da fisiologia cardiovascular. Entretanto, está indicado quando se observa a fração de ejeção de $<40 \%$ à ecocardiografia bidimensional ou em pacientes que necessitam dopamina acima de $10 \mu \mathrm{g} / \mathrm{kg} /$ min, dois vasopressores onde um não seja vasopressina (fármaco de escolha onde dose máxima não deve ultrapassar 2,4 U/h) ou nos casos de persistência da instabilidade cardiovascular. Chama-se atenção que a dopamina não é o vasopressor de escolha, nem mesmo de segunda linha onde ocupam este lugar a norepinefrina, epinefrina ou fenilefrina, onde doses acima de $0,2 \mu \mathrm{g} / \mathrm{kg} / \mathrm{min}$ devem ser usados com muita cautela. Recomenda-se PCPE 6-10, Cl > 2,4 L/min- $\mathrm{m}^{2}$, RVS 800-1200 dinas/sec-seg ${ }^{5}$.

\section{Controle Glicêmico e Nutrição}

Recomenda-se controle glicêmico (alvo de 4-8 mmol/ L) através da infusão contínua de insulina. Não se deve interpretar esta condição como contra-indicação para doação de células das ilhotas de Langerhans, onde houver qualquer suspeita, os níveis de hemoglobina A1C devem ser aferidos ${ }^{45-47}$.

Suporte calórico protéico deve ser realizado com solução glicosada, por via venosa e alimentação enteral que é interrompida ao momento da entrada no centro cirúrgico. Não se indica nutrição parenteral (adaptado das rotinas do Trillium Gift of Life, Toronto, Canadá, agência provincial de captação de órgãos).

\section{Diabetes Insipidus e Distúrbios Hidroeletrolíticos Ácido-Base}

Diabetes insipidus pode ser definido como débito urinário acima de $4 \mathrm{~mL} / \mathrm{kg} / \mathrm{h}$, associado à sódio sérico > $145 \mathrm{mmol} / \mathrm{L}$ e osmolaridade $>300 \mathrm{mosM}$ com osmolaridade urinária $<200$ mosM. Para diagnóstico diferencial leva-se em conta os dados laboratoriais como demonstrado na tabela $3^{1,3,6,13,17,19}$.

Vasopressina é o tratamento de escolha em especial se associado à instabilidade hemodinâmica, suas doses usuais compreendem 0,25 a $1 \mu \mathrm{g}$ a cada 6 horas para crianças ou $1 \mathrm{a} 4 \mu \mathrm{g}$, seguido de 1 a $2 \mu \mathrm{g}$ a cada 6 horas para adultos. O objetivo é manter a natremia entre 130 e $150 \mathrm{mmol} / \mathrm{L}$ e débito urinário entre 0,5 e 3 $\mathrm{mL} / \mathrm{kg} / \mathrm{h}^{1,3,6,13,17,19}$.

Níveis séricos de sódio, cálcio, potássio, fosfato e magnésio devem ser acompanhados e normalizados ${ }^{1,3,6,13,17,19}$.

\section{Terapia Hormonal Combinada}

A terapia hormonal combinada é definida como o uso de hormônio tireoidiano, vasopressina e metilprednisolona. Recomenda-se a sua utilização em todos os doadores com fração de ejeção de VE < $40 \%$ ao ecocardiograma 2D, entretanto sua utilização nos demais doadores exige considerações (adaptado das rotinas do Trillium Gift of Life, Toronto, Canadá, agência provincial de captalcão de órgãos).

Dados da UNOS obtidos de um grande estudo retrospectivo com 18.726 pacientes com morte cerebral sugerem um substancial benefício obtido com este tratamento com um mínimo de risco com maior aproveitamento de órgãos além de maior sobrevida dos enxertos. A utilização de corticóide é defendida por todos os centros transplantadores de pulmão pelos seus efeitos imunomodulatórios ${ }^{48-53}$.

Tabela 3 - Dados Laboratoriais no Diagnóstico Diferencial da Diabetes Insipidus.

\begin{tabular}{lccc}
\hline & Diabetes Insipidus & Manitol & Ambos \\
\hline Osmolalidade & $>292 \mathrm{mosm} / \mathrm{kg}$ & $>292 \mathrm{mosm} / \mathrm{kg}$ & $>292 \mathrm{mosm} / \mathrm{kg}$ \\
Gap osmolar & $5-10 \mathrm{mosm} / \mathrm{kg}$ & $10 \mathrm{mosm} / \mathrm{kg}$ & $>10 \mathrm{mosm} / \mathrm{kg}$ \\
Débito urinário & $>4 \mathrm{~mL} / \mathrm{kg}$ & $>4 \mathrm{~mL} / \mathrm{kg}$ & $>4 \mathrm{~mL} / \mathrm{kg}$ \\
Na sérico & $>150 \mathrm{mmol} / \mathrm{l}$ & $>150 \mathrm{mmol} / \mathrm{l}$ & $>150 \mathrm{mmol} / \mathrm{l}$ \\
Na urinário & $>10 \mathrm{mmol} / \mathrm{l}$ & $50-70 \mathrm{mmol} / \mathrm{l}$ & $10-50 \mathrm{mmol} / \mathrm{l}$ \\
Gravidade específica & $<1010$ & 1020 & $<1010-1020$ \\
Osmolalidade U & $<300 \mathrm{mosm}$ & $=$ Osmolalidade soro & Variável \\
\hline
\end{tabular}

Adaptado do Programa de Transplante de Pulmão de Toronto, Universidade de Toronto (2006) 


\section{Transfusões}

As seguintes condutas diante dos níveis de hemoglobina, plaquetas e parâmetros de coagulação são sugeridas $^{38,54,55}$.

- Hemoglobina entre 90 e 100 g/L é o nível mais apropriado para melhorar as funções cardiopulmonares. Entretanto, os níveis limítrofes de $70 \mathrm{~g} / \mathrm{L}$ correspondem aos níveis mais baixos na manutenção de doadores de órgãos. Efeitos adversos incluem a ativação inflamatória sistêmica especialmente relacionada à idade do sangue estocado. Em especial nos doadores de pulmão deve-se considerar a reposição criteriosa com cristalóide não Ringer. Nos casos onde se faça necessária a transfusão dá-se preferência ao sangue depletado de leucócitos para evitar a contaminação pelo citomegalovírus;

- Não há limites definidos para número de plaquetas, INR ou PTT sendo o paciente tratado de acordo com a apresentação clínica;

- Sangue para testes sorológicos deve ser colhido antes de eventual transfusão, sempre que possível.

\section{Estudos Microbiológicos e Antibioticoterapia Pro- filática}

Culturas de sangue positivas não são contra-indicações a doação de órgãos; entretanto, antibioticoterapia apropriada deve ser iniciada nos casos de infecção presumida ou comprovada. Até a presente data não existe um tempo mínimo para tratamento anterior a captação de órgãos que possa ser prevista com segurança. Culturas sangüíneas devem ser repetidas diariamente (adaptado das rotinas do Trillium Gift of Life, Toronto, Canadá, agência provincial de captação de órgãos).

\section{RECOMENDAÇÕES ÓRGÃO-ESPECÍFICAS}

\section{Coração}

Como já referido, a avaliação mínima consiste em ECG e ecocardiografia bidimensionais, esse último após a reanimação do potencial doador. Caso a fração de ejeção esteja menor que $40 \%$ a instalação de monitorização invasiva está indicada. Este cateter não se destina a monitorização exclusiva e sim avaliar a função cardiopulmonar e fornecer informações para decisão adequada quanto a aceitabilidade destes órgãos ${ }^{37,38}$. Monitorização sorológica também constitui um instrumento importante sendo a troponina I ou T medidas a cada 12 horas $^{37,38}$.

Quando possível, a coronariografia está sempre indicada. Casos especiais são aqueles onde a idade do doador é avançada (masculino $>55$ ou feminino $>60$ anos), presença de mais de 3 fatores de risco (tabagismo, hipertensão arterial, diabetes, hiperlipidemia, massa corpórea $>32$, história familiar, história de doença coronariana, isquemia no ECG, anormalidades na parede anterior à ecocardiografia e baixa fração de ejeção) para doença coronariana ou história de uso de cocaína. A utilização de enxerto com doença coronária, varia de centro para centro onde a realização de intervenções percutâneas ou bypass coronariano é julgado caso-a-caso ${ }^{37,38,56-58}$.

\section{Pulmão}

Oximetria de pulso, gases arteriais seriados, aspiração de tubo traqueal, broncoscopia e lavado bronco-alveolar, radiografia de tórax são rotinas estabelecidas na maioria dos centros transplantadores. $\mathrm{FiO}_{2}$ deve ser regulada para garantir a $\mathrm{SO}_{2}>95 \%$ e a $\mathrm{PaO}_{2}>80, \mathrm{VC}$ 8-10 $\mathrm{cmH}_{2} \mathrm{O}$, PEEP ideal seria em torno de $5 \mathrm{mmHg}$ com $\mathrm{PaCO}_{2} 35$ a $45 \mathrm{mmHg}^{29,31-34,37,55,56}$.

Rotação do paciente a cada duas horas pode ser interessante, especialmente nos casos de difícil oxigenação, associado a intensificação de aspiração traqueal, fisioterapia e manobras de recrutamento alveolar (PEEP $15 \mathrm{mmHg}$ por 30 segundos ou PIP de $30 \mathrm{mmHg}$ por 30 segundos).

Não existem limites inferiores de $\mathrm{PaO}_{2}$ que precludam a utilização dos pulmões em situações onde o bom senso predomina; entretanto, nos casos de doação unilateral (lesão unilateral de pulmão) de pulmão $\mathrm{PvO}_{2}$ deve ser avaliada para se certificar da boa função contra-lateral (adaptado do Programa de Transplante de Pulmão de Toronto, 2007) $)^{32,40,41}$.

Ventilação protetora, ou seja, baixos volumes-correntes é benéfica para pacientes portadores de SARA (síndrome da angústia respiratória aguda), porém não existem dados específicos para doadores de órgãos ${ }^{32,59}$.

Com a broncoscopia e o lavado bronco-alveolar terse-ão informações significativas quanto ao estado séptico do pulmão. O exame de Gram ou cultura deve nortear o tratamento antibioticoterápico. A antibioticoterapia profilática é praticada nos casos de alto risco para broncopneumonia, entretanto não existem dados comprovando a sua utilidade, lembrando que antibióticos nefrotóxicos devem ser evitados ${ }^{60-63}$.

\section{Fígado}

Doadores de fígado em potencial devem ser acessados quanto a história de icterícia, hepatite, alcoolismo. Deve ter aferidos os níveis de TGO (transaminase glutâmico 
oxalacética), TGP (transaminase glutâmica pirúvica), fosfatase alcalina, GGT (gama glutamil transferase), INR (Relação Internacional Normalizada) e tempo de protrombina a cada 6 h. A biópsia hepática encontra-se indicada nos casos de peso acima de $100 \mathrm{~kg}$ ou índice de massa corpórea $>30$ ou no caso de positividade de anticorpo contra vírus da hepatite $\mathrm{C}$ (adaptado do Programa de Transplante de Pulmão de Toronto, 2007).

\section{Rim}

Os rins são geralmente considerados próprios para doação quando apresenta depuração de creatinina superior a $80 \mathrm{~mL} / \mathrm{min} / 1,73 \mathrm{~m}^{2}$ e sedimentoscopia normal. De forma semelhante ao aplicado a avaliação hepática não é exigência da realização de ultra-som abdominal renal pré-operatório. Certas situações exigem avaliação histopatológica do parênquima renal, são elas: idade acima de 65 anos ou, creatinina acima de 133 $\mu \mathrm{mol} / \mathrm{L}$, hipertensão, diabetes ou urinálise anormal ${ }^{64}$.

\section{EXPANDINDO AS POSSIBILIDADES DE DOAÇÃO DE ÓRGÃOS}

Não seria possível finalizar um estudo como este sem abordar dois temas bastante importantes no contexto da doação de órgãos a doação intervivos e a doação depois da parada cardíaca. Ambas as abordagens são variantes destinadas a compensar a escassez de doadores.

A doação intervivos é bastante aplicada no transplante renal, hepático, pulmonar e cardíaco quando se é aceito o chamado transplante dominó, ou seja, o transplante do bloco coração pulmão para um receptor cujo coração saudável que é transplantado para um terceiro paciente. Apresentam importantes características que os tornam muito convenientes como: maior compatibilidade HLA em potencial, menor tempo de isquemia do enxerto e possibilita o transplante em melhores condições clínicas já o que receptor não é obrigado a enfrentar longas filas de espera ${ }^{21}$.

O número de doadores convencionais vem diminuindo em razão de dois fatores principais: o menor número de pacientes vem a falecer de trauma e eventos cérebrovasculares em virtude dos significativos progressos na área de terapia intensiva e pelo fato de que os meios diagnósticos tornam-se cada vez mais específicos diminuindo o número de pacientes que se encaixam nas especificações. A parada cardiocirculatória sobrevêm a retirada do suporte nas unidades de terapia intensiva em parcela significativa de pacientes que não atendem aos critérios de morte encefálica. A utilização destes órgãos é dependente da extensão do tempo da chamada isquemia morna, isto é, o tempo até que o órgão seja irrigado com solução de preservação. Apenas aqueles classificados como categoria 3 ou 4, segundo a classificação de Maastricht (Quadro 3) estariam aptos para doação de órgãos ${ }^{10,65,66}$. Um aprofundame nto nas técnicas envolvidas ultrapassa os propósitos desta revisão e o leitor interessado poderá encontrar uma extensa lista de referências nos portais de pesquisa médica.

Quadro 3 - Classificação de Maastricht (1995) para Doadores após Parada Cardíaca.

\begin{tabular}{l}
\hline Categoria 1 Morto a chegada ao Hospital \\
Categoria 2 Reanimação sem sucesso \\
Categoria 3 Parada cardíaca aguardada \\
Categoria 4 Parada cardíaca em doador com morte encefálica \\
Categoria 5 Parada cardíaca não esperada em paciente crítico
\end{tabular}

Em conclusão com o aumento significativo das listas de espera por órgãos e a escassez de órgãos disponíveis leva-nos a um esforço para aprimorar as técnicas existentes de captação e preservação assim como desenvolver novas medidas para seu aproveitamento de forma a reduzir a mortalidade nas filas de espera que são sempre uma sombra nos programas de transplantes.

\section{REFERÊNCIAS}

01. Tuttle-Newhall JE, Collins BH, Kuo PC et al - Organ donation and treatment of the multi-organ donor. Curr Probl Surg. 2003;40:266-310.

02. Joost $A B$, Ploeg RJ - Effects of brain death on donor viability. Curr Opin Organ Transplant. 2001;6:75-82.

03. Elliot JM - Brain death. Trauma. 2003;5:23-42.

04. Committee of the Harvard Medical School. Brain Death. Harvard Medical School. 1969.

05. Siebels $\mathrm{M}$, Theodorakis J, Schmeller $\mathrm{N}$ et al - Risks and complications in 160 living kidney donors who underwent nephroureterectomy. Nephrol Dial Transplant. 2003;18:2648-2654.

06. Powner DJ, Kellum JA, Darby JM - Abnormalities in fluids, eletrolytes, andmetabolism of organ donors. Prog Transpl. 2000;10:88-94.

07. de Perrot M, Keshavjee S - Lung preservation. Ann Thorac Surg, 2002;74:629-631.

08. de Perrot M, Liu M, Waddell TK et al - Ischemia-reperfusion-induced lung injury. Am J Respir Crit Care Med. 2003;167:490-511.

09. de Perrot M, Keshavjee S - Lung transplantation. Lung preservation. Chest Surg Clin N Am, 2003;13:443-462.

10. Egan TM - Non-heart-beating donors in thoracic transplantation. J Heart Lung Transplant. 2004;23:3-10.

11. Lloyd-Jones $\mathrm{H}$ - Attitudes of nurses to donor organ retrieval and visiting surgical teams. The Papworth experience. Br J Theatre Nurs, 1996;5:28-31.

12. Lloyd-Jones H, Wheeldon DR, Smith JA et al - An approach to the retrieval of thoracic organs for transplantation. AORN J, 1996;63:416-423.

13. Booij LH - Brain death and care of the brain death patient. Curr Anaesth Crit Care, 1999;10:312-318.

14. Wijdicks EF - The diagnosis of brain death. N Engl J Med, 2001;344:12151221.

15. Avlonitis VS, Fisher AJ, Kirby JA et al - Pulmonary transplantation: the role of brain death in donor lung injury. Transplantation. 2003;75:1928-1933. 
16. Boscoe MJ, Goodwin AT, Amrani M et al - Endothelins and the lung. Int J Biochem Cell Biol, 2000;32:41-62.

17. Ramos HC, Lopez R - Critical care management of the brain-dead organ donor. Curr Opin Organ Transplant, 2002;7:70-75.

18. Snell Gl, Griffiths A, Macfarlane L et al - Maximizing thoracic organ transplant opportunities: the importance of efficient coordination. J Heart Lung Transplant. 2000;19:401-407.

19. MacLean A, Dunning J - The retrieval of thoracic organs: donor assessment and management. Br Med Bull, 1997;53:829-843.

20. Wight $\mathrm{C}$, Cohen $\mathrm{B}$ - Organ shortages: maximising the donor potential. $\mathrm{Br}$ Med Bull. 1997:53:817-828.

21. Ridley S, Bonner S, Bray K et al - UK guidance for non-heart-beating donation. Br J Anaesth, 2005;95:592-595.

22. Orens JB, Boehler $\mathrm{A}$, de Perrot $\mathrm{M}$ et al $-\mathrm{A}$ review of lung transplant donor acceptability criteria. J Heart Lung Transplant, 2003;22:1183-1200.

23. Aigner $\mathrm{C}$, Jaksch $\mathrm{P}$, Taghavi $\mathrm{S}$ et al - Donor total lung capacity predicts recipient total lung capacity after size-reduced lung transplantation. J Heart Lung Transplant, 2005;24:2098-2102.

24. Aigner C, Winkler G, Jaksch P et al - Size-reduced lung transplantation: an advanced operative strategy to alleviate donor organ shortage. Transplant Proc, 2004;36:2801-2805.

25. Aigner $\mathrm{C}$, Mazhar S, Jaksch $\mathrm{P}$ et al - Lobar transplantation, split lung transplantation and peripheral segmental resection--reliable procedures for downsizing donor lungs. Eur J Cardiothorac Surg, 2004;25:179-183.

26. Trow TK - Clinical year in review I: diagnostic imaging, asthma, lung transplantation, and interventional pulmonology. Proc Am Thorac Soc, 2006;3:553-556.

27. Langenbach SY, Zheng L, McWilliams T - Airway vascular changes after lung transplant: potential contribution to the pathophysiology of bronchiolitis obliterans syndrome. J Heart Lung Transplant, 2005;24:15501556.

28. Oto T, Griffiths A, Levvey B et al - Donor history of asthma is not a contraindication to lung transplantation: 12-year single-center experience. J Heart Lung Transplant, 2004;23:309-316.

29. Botha P, Fisher AJ, Dark JH - Marginal lung donors: A diminishing margin of safety? Transplantation, 2006;82:1273-1279.

30. Hornby K, Ross H, Keshavjee S et al - Non-utilization of hearts and lungs after consent for donation: a Canadian multicentre study. Can J Anaesth, 2006;53:831-837.

31. Kutsogiannis DJ, Pagliarello G, Doig $C$ et al - Medical management to optimize donor organ potential: review of the literature. Can J Anaesth, 2006:53:820-830.

32. Filosso PL, Turello D, Cavallo A et al - Lung donors selection criteria: a review. J Cardiovasc Surg (Torino), 2006;47:361-366.

33. Botha P, Trivedi D, Weir CJ et al - Extended donor criteria in lung transplantation: impact on organ allocation. J Thorac Cardiovasc Surg, 2006;131:1154-1160.

34. Pierre AF, Sekine $\mathrm{Y}$, Hutcheon MA et al - Marginal donor lungs: a reassessment. J Thorac Cardiovasc Surg, 2002;123:421-428.

35. Stoica SC, Satchithananda DK, Charman S et al - Swan-Ganz catheter assessment of donor hearts: outcome of organs with borderline hemodynamics. J Heart Lung Transplant, 2002;21:615-622.

36. Follette DM, Rudich SM, Babcock WD - Improved oxygenation and increased lung donor recovery with high-dose steroid administration after brain death. J Heart Lung Transplant, 1998;17:423-429.

37. Delgado DH, Rao V, Ross HJ - Donor management in cardiac transplantation. Can J Cardiol, 2002;18:1217-1223.

38. Van Bakel AB - The cardiac transplant donor: identification, assessment, and management. Am J Med Sci, 1997;314:153-163.

39. Bittner HB, Kendall SW, Chen EP et al - The combined effects of brain death and cardiac graft preservation on cardiopulmonary hemodynamics and function before and after subsequent heart transplantation. $J$ Heart Lung Transplant, 1996;15:764-777.

40. Pennefather SH, Bullock RE, Dark JH - The effect of fluid therapy on alveolar arterial oxygen gradient in brain-dead organ donors. Transplantation, 1993;56:1418-1422.

41. Pennefather SH, Bullock RE, Mantle D et al - Use of low dose arginine vasopressin to support brain-dead organ donors. Transplantation, 1995;59:58-62.

42. Dujardin KS, McCully RB, Wijdicks EF et al - Myocardial dysfunction associated with brain death: clinical, echocardiographic, and pathologic features. J Heart Lung Transplant, 2001;20:350-357.
43. Chen JM, Cullinane S, Spanier TB et al - Vasopressin deficiency and pressor hypersensitivity in hemodynamically unstable organ donors. Circulation, 1999;100:(Suppl19):II244-II246.

44. Otero RM, Nguyen HB, Huang DT et al - Early goal-directed therapy in severe sepsis and septic shock revisited: concepts, controversies, and contemporary findings. Chest, 2006;130:1579-1595.

45. O'Gorman D, Kin T, Murdoch T et al - The standardization of pancreatic donors for islet isolation. Transplant Proc, 2005;37:1309-1310.

46. Hesse UJ, Berrevoet F, Pattyn P et al - Donor parameters of pancreas grafts processed for islet or beta-cell isolation and transplantation. Transplant Proc, 1997;29:2259.

47. Masson F, Thicoipe M, Gin H et al - The endocrine pancreas in braindead donors. A prospective study in 25 patients. Transplantation, 1993;56:363-367.

48. Novitzky D, Cooper DK, Rosendale JD et al - Hormonal therapy of the brain-dead organ donor: experimental and clinical studies. Transplantation, 2006;82:1396-1401.

49. Maghnie M, Uga E, Temporini F et al - Evaluation of adrenal function in patients with growth hormone deficiency and hypothalamic-pituitary disorders: comparison between insulin-induced hypoglycemia, low-dose ACTH, standard ACTH and CRH stimulation tests. Eur J Endocrinol, 2005:152:735-741.

50. Agha A, Rogers B, Sherlock $\mathrm{M}$ et al - Anterior pituitary dysfunction in survivors of traumatic brain injury. J Clin Endocrinol Metab, 2004;89:4929-4936.

51. Agha A, Rogers B, Mylotte D et al - Neuroendocrine dysfunction in the acute phase of traumatic brain injury. Clin Endocrinol, 2004;60:584-591.

52. Mangieri P, Suzuki K, Ferreira ML et al - Evaluation of pituitary and thyroid hormones in patients with subarachnoid hemorrhage due to ruptured intracranial aneurysm. Arq Neuropsiquiatr, 2003;61:14-19

53. Mitchell DH, Owens B - Replacement therapy: arginine vasopressin (AVP), growth hormone (GH), cortisol, thyroxine, testosterone and estrogen. J Neurosci Nurs. 1996;28:140-152.

54. Zaroff JG, Rosengard BR, Armstrong WF et al - Consensus conference report: maximizing use of organs recovered from the cadaver donor: cardiac recommendations, March 28-29, 2001, Crystal City, Va. Circulation, 2002;106:836-841.

55. Hebert PC, Wells G, Blajchman MA et al - A multicenter, randomized, controlled clinical trial of transfusion requirements in critical care. Transfusion Requirements in Critical Care Investigators, Canadian Critical Care Trials Group. N Engl J Med, 1999;340:409-417.

56. McGiffin DC, Savunen T, Kirklin JK et al - Cardiac transplant coronary artery disease. A multivariable analysis of pretransplantation risk factors for disease development and morbid events. J Thorac Cardiovasc Surg, 1995;109:1081-1089.

57. Costanzo MR, Naftel DC, Pritzker MR et al - Heart transplant coronary artery disease detected by coronary angiography: a multiinstitutional study of preoperative donor and recipient risk factors. Cardiac Transplant Research Database. J Heart Lung Transplant, 1998;17:744-753.

58. Kirklin JK, Naftel DC, Levine TB et al - Cytomegalovirus after heart transplantation. Risk factors for infection and death: a multiinstitutional study. The Cardiac Transplant Research Database Group. J Heart Lung Transplant, 1994;13:394-404.

59. Taskar V, John J, Evander E et al - Healthy lungs tolerate repetitive collapse and reopening during short periods of mechanical ventilation. Acta Anaesthesiol Scand, 1995;39:370-376.

60. Low DE, Kaiser LR, Haydock DA et al - The donor lung: infectious and pathologic factors affecting outcome in lung transplantation. $J$ Thorac Cardiovasc Surg, 1993;106:614-621.

61. Bhorade SM, Vigneswaran W, McCabe MA et al - Liberalization of donor criteria may expand the donor pool without adverse consequence in lung transplantation. J Heart Lung Transplant, 2000;19:1199-1204.

62. Zibari GB, Lipka J, Zizzi $\mathrm{H}$ et al - The use of contaminated donor organs in transplantation. Clin Transplant, 2000;14:397-400.

63. Aigner $\mathrm{C}$, Winkler $\mathrm{G}$, Jaksch $\mathrm{P}$ et al - Extended donor criteria for lung transplantation--a clinical reality. Eur J Cardiothorac Surg, 2005;27:757-761.

64. Port FK, Bragg-Gresham JL, Metzger RA et al - Donor characteristics associated with reduced graft survival: an approach to expanding the pool of kidney donors. Transplantation, 2002;74:1281-1286.

65. Corris PA - Non-heart beating lung donation: aspects for the future. Thorax, 2002;57:(Supp12):II53-II56.

66. Steen S, Sjoberg T, Pierre L et al - Transplantation of lungs from a nonheart-beating donor. Lancet, 2001;357:825-829. 